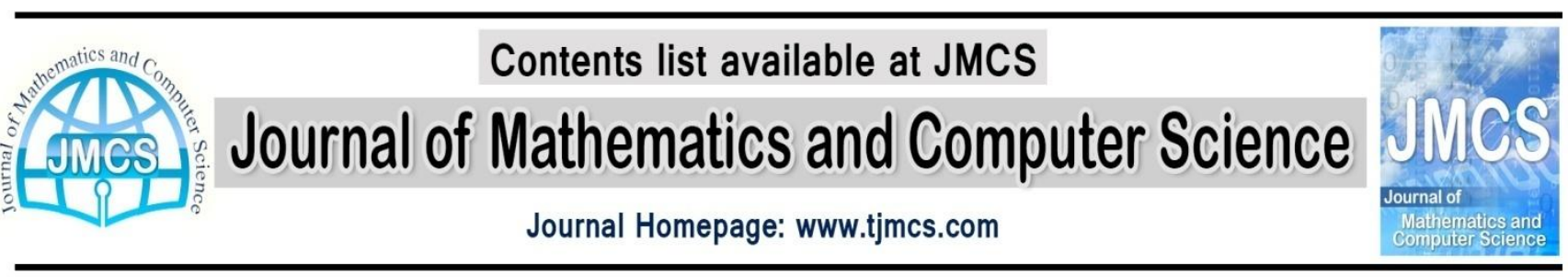

\title{
New Implementation of Reproducing Kernel Hilbert Space Method for Solving a Class of Third-order Differential Equations
}

\author{
Eslam Moradi ${ }^{1{ }^{*},}$, Aasadolla Yusefi ${ }^{1}$, Abolfazl Abdollahzadeh², Elham Tila ${ }^{3}$ \\ ${ }^{1}$ Department of Mathematics, Faculty of Mathematical Sciences and Computer, Kharazmi \\ University, 50 Taleghani Avenue, 1561836314 Tehran, Iran \\ ${ }^{2}$ Department of Mathematics, Faculty of Mathematical Sciences and Statistics, Birjand \\ University, Birjand, Iran \\ ${ }^{3}$ Department of Mathematics Sciences and Statistics, Islamic Azad University, Dezful \\ Branch, Dezful, Iran
}

*E-mail: eslam.moradi@ gmail.com (Eslam Moradi; The corresponding author)

\section{Article history: \\ Received July 2014 \\ Accepted August 2014 \\ Available online August 2014}

\begin{abstract}
In this paper, we apply the new implementation of reproducing kernel Hilbert space method to give the approximate solution to some third-order boundaryvalue problems with variable coefficients. In this method, the analytical solution is expressed in the form of a series. At the end, two examples are given to illustrate implementation, accuracy and effectiveness of the method.
\end{abstract}

Keywords: Reproducing kernel Hilbert space method; Boundary value problems; Third-order differential equations; Approximatesolution.

\section{Introduction}

Reproducing kernel Hilbert space method is a promising method which has beenapplied more and more for solving various problems such asordinary differential equations, partial differential equations, differential-difference equations, integral equations, and etc. inthe previous decades [1]-[22]. Approximate solutionof the Fredholm integral equation of the first kind in the 
reproducing kernel Hilbert space was presented by Du and Cui [3,4], solution of a system of linear Volterraintegral equations was discussed by Yang et al. [5],solvability of a class of Volterra integral equations with weaklysingular kernel using reproducing kernel Hilbert space method were investigated in [6,7,8],Geng[9] explained how to solve the Fredholm integralequation of the third kind in the reproducing kernel Hilbertspace method. These are a bunch of extensive works related to reproducing kernel Hilbertspace method for solving integral equations.

In 1986, Cui Minggen[10] gave the reproducing kernel space $W_{2}^{1}[a, b]$ and its reproducing kernel. This technique has successfully been treated singular linear two-point boundary value problems [11,12],singular nonlinear second-order boundary value problems $[13,14,15,16]$, nonlinear system of boundary value problems [17],third-order boundary value problems [18,19], fifth-order boundary value problems [20], and nonlinear partialdifferential equations [21] in recent years.

This paper investigates the approximate solution of the following third-order boundary value problem using new implementation of reproducing kernel Hilbertspace method

$$
\left\{\begin{array}{c}
y^{\prime \prime \prime}(x)+p(x) y(x)=f(x), \quad 0 \leq x \leq 1, \\
y(0)=A, y^{\prime}(0)=B, \quad y^{\prime}(1)=C,
\end{array}\right.
$$

where $p(x), f(x)$ are analytical known functions defined on theinterval $[0,1]$, unknown function $y(x)$ is continuouson the interval $[0,1]$ and $A, B, C$ are finite real constants.

Several numerical techniques have been proposed to solve high-order differential equations $[23,24,25]$.

As we known, Gram-Schmidt orthogonalization process isnumerically unstable and in addition it may take a lot oftime to produce numerical approximation. Here, instead ofusing orthogonal process, we successfully make use of thebasic functions which are obtained by reproducing kernel Hilbertspace method.

This paper is organized as follows. In thefollowing section, we introduce some useful definitions and theorems. Section 3 is devoted to solve Eq. (1) by new implementation of reproducing kernel Hilbert space method. Two numerical examples are presented in Section 4. We end the paper with a few conclusions.

\section{Reproducing Kernel Spaces}

In this section, we follow the recent work of [1]-[22] and represent some useful materials.

\section{Definition 1.}

Let $\left(\mathcal{H},<\ldots,>_{\mathcal{H}}\right)$ be a Hilbert space of real-valued functions on some nonempty set $\mathcal{X}$. A functionk: $\mathcal{X} \times \mathcal{X} \rightarrow \mathbb{R}$ is said to be the reproducing kernel of $\mathcal{H}$ if and only if

1. $k(x,.) \in \mathcal{H}, \quad \forall x \in \mathcal{X}$,

2. $<\varphi(),. k(x,)>._{\mathcal{H}}=\varphi(x), \forall \varphi \in \mathcal{H}, \forall x \in \mathcal{X}, \quad$ (reproducing property). 
It is known that the reproducing kernel of a reproducing kernel Hilbertspace is unique and the existence of a reproducing kernel is according to the Riesz Representation Theorem. The reproducing kernel $k$ of a Hilbert space $\mathcal{H}$ quite determines the space $\mathcal{H}$. Each set of functions $\left\{\varphi_{i}\right\}_{i=1}^{\infty}$ which converges strongly to a function $\varphi$ in $\mathcal{H}$, converges also in the pointwise sense. In addition, this convergence is uniform on every subset of $\mathcal{X}$ on which $x \mapsto k(x, x)$ is bounded.

\section{Definition 2.}

$$
\begin{gathered}
W_{2}^{4}[0,1]=\left\{y(x) \mid y^{\prime \prime \prime}(x) \text { is an absolute continuous real valued function and } y^{(4)}(x)\right. \\
\left.\in L^{2}[0,1], \quad y(0)=y^{\prime}(0)=y^{\prime}(1)=0\right\}
\end{gathered}
$$

The inner product and the norm in the function space $W_{2}^{4}[0,1]$ are defined as follows.

$$
\left\{\begin{array}{l}
\langle u, v\rangle_{W_{2}^{4}}=u^{\prime \prime}(0) v^{\prime \prime}(0)+\int_{0}^{1} u^{(4)}(x) v^{(4)}(x) d x, \\
\|\mathrm{u}\|_{W_{2}^{4}}=\sqrt{\langle u, u\rangle_{W_{2}^{4}}} .
\end{array}\right.
$$

Let's assume that function $R(x, t) \in W_{2}^{4}[0,1]$ satisfies the following generalized differential equations

$$
\left\{\begin{array}{c}
\frac{\partial^{8} R(x, t)}{\partial t^{8}}=\delta(t-x), \\
\mathrm{R}(\mathrm{x}, 1)-\frac{\partial^{7} R(x, 1)}{\partial t^{7}}=0, \\
\frac{\partial^{4} R(x, 1)}{\partial t^{4}}=0, \quad \frac{\partial^{4} R(x, 0)}{\partial t^{4}}, \\
\frac{\partial^{5} R(x, 1)}{\partial t^{5}}=0, \quad \frac{\partial^{5} R(x, 0)}{\partial t^{5}} .
\end{array}\right.
$$

where $\delta$ is the Dirac delta function. Therefore, the following theorem holds.

Theorem 1.Under the assumptions of Eq. (2), Hilbert space $W_{2}^{4}[0,1]$ isa reproducing kernel Hilbertspace with the reproducing kernel function $R(x, t)$, namely for any $y(t) \in W_{2}^{4}[0,1]$ and each fixed $x \in[0,1]$, there exists $R(x, t) \in W_{2}^{4}[0,1], t \in[0,1]$, such that

$$
<y(.), R(x, .)>_{W_{2}^{4}}=y(x) \text {. }
$$

While $x \neq t$, function $R(x, t)$ is the solution of the followingconstant linear homogeneous differential equation with 8 orders,

$$
\frac{\partial^{8} R(x, t)}{\partial t^{8}}=0
$$

with the boundary conditions: 


$$
\left\{\begin{array}{cl}
\mathrm{R}(\mathrm{x}, 1)-\frac{\partial^{7} R(x, 1)}{\partial t^{7}}=0, \\
\frac{\partial^{4} R(x, 1)}{\partial t^{4}}=0, & \frac{\partial^{4} R(x, 0)}{\partial t^{4}}, \\
\frac{\partial^{5} R(x, 1)}{\partial t^{5}}=0, & \frac{\partial^{5} R(x, 0)}{\partial t^{5}} .
\end{array}\right.
$$

We know that Eq. (3) has characteristic equation $\lambda^{8}=0$, and theeigenvalue $\lambda=0$ is a root whose multiplicity is 8. Hence, the general solution of Eq. (2) is

$$
R(x, t)= \begin{cases}\sum_{i=1}^{8} c_{i}(x) t^{i-1}, & t \leq x, \\ \sum_{i=1}^{8} d_{i}(x) t^{i-1}, & t>x .\end{cases}
$$

Now, we are ready to calculate the coefficients $c_{i}(x)$ and $d_{i}(x), i=1, \ldots, 8$. Since

$$
\frac{\partial^{8} R(x, t)}{\partial t^{8}}=\delta(t-x)
$$

we have

$$
\left\{\begin{array}{l}
\frac{\partial^{\mathrm{k}} \mathrm{R}\left(\mathrm{x}, \mathrm{x}^{+}\right)}{\partial \mathrm{t}^{\mathrm{k}}}=\frac{\partial^{\mathrm{k}} \mathrm{R}\left(\mathrm{x}, \mathrm{x}^{-}\right)}{\partial \mathrm{t}^{\mathrm{k}}}, \\
\frac{\partial^{7} \mathrm{R}\left(\mathrm{x}, \mathrm{x}^{+}\right)}{\partial \mathrm{t}^{\mathrm{k}}}-\frac{\partial^{7} \mathrm{R}\left(\mathrm{x}, \mathrm{x}^{-}\right)}{\partial \mathrm{t}^{\mathrm{k}}}=1 .
\end{array}\right.
$$

Then, using Eqs. (4) and (6), the unknown coefficients of Eq. (5) are uniquely obtained (in Apendix A).

\section{Definition 3.}

$W_{2}^{1}[0,1]$

$=\left\{y(x) \mid y(x)\right.$ is an absolute continuous real valued function on the interval $[0,1]$ and $y^{\prime}(x)$ $\left.\in L^{2}[0,1]\right\}$.

The inner product and the norm in the function space $W_{2}^{1}[0,1]$ are defined as follows.

$$
\left\{\begin{array}{c}
\langle u, v\rangle_{W_{2}^{1}}=u(0) v(0)+\int_{0}^{1} u^{\prime}(x) v^{\prime}(x) d x, \\
|| \mathrm{u} \|_{W_{2}^{1}}=\sqrt{\langle u, u\rangle_{W_{2}^{1}}} .
\end{array}\right.
$$

Theorem 2.Hilbert space $W_{2}^{1}[0,1]$ is a reproducing kernel space with the reproducing kernel function 


$$
Q(x, t)= \begin{cases}1+\mathrm{t}, & t \leq x \\ 1+\mathrm{x}, & t>x\end{cases}
$$

that is, for any $y(t) \in W_{2}^{1}[0,1]$ and each fixed $x \in[0,1]$, it follows that

$$
<y(.), Q(x, .)>_{W_{2}^{1}}=y(x) \text {. }
$$

\section{Reproducing Kernel Hilbert Space Method}

We suppose that Eq. (1) has a unique solution. To deal with the system, we consider Eq. (1) as

$$
\mathbb{L} y(x)=f(x), \quad 0 \leq x \leq 1,
$$

where $\mathbb{L} y(x)=y^{\prime \prime \prime}(x)+p(x) y(x)$, it is clear that $\mathbb{L}$ is the bounded linear operator of $W_{2}^{4}[0,1] \rightarrow W_{2}^{1}[0,1]$. We shall give the representation of analytical solution of Eq. (7) in the space $W_{2}^{4}[0,1]$. Set $\varphi_{i}(x)=Q\left(x_{i}, x\right)$ and $\psi_{i}(x)=\mathbb{L}^{*} \varphi_{i}(x), i=1,2, \ldots$, where $Q\left(x_{i}, x\right)$ is the reproducing kernel of $W_{2}^{1}[0,1]$ and $\mathbb{L}^{*}$ is the adjoint operator of $\mathbb{L}$.

Theorem 3.Let $\left\{x_{i}\right\}_{i=1}^{\infty}$ be a dense subset of interval $\$[0,1] \$$, then $\left\{\psi_{i}(x)\right\}_{i=1}^{\infty}$ is a complete system of $W_{2}^{4}[0,1]$ and $\psi_{i}(x)=\left.\mathbb{L}_{t} R(x, t)\right|_{t=x_{i}}$, where the subscript $t$ in the operator $\mathbb{L}$ indicates that the operator Lapplies to the functionof $t$.

Usually, a normalized orthogonal system is constructed from $\left\{\psi_{i}(x)\right\}_{i=1}^{\infty}$ by using the GramSchmidt algorithm, and then the approximate solution be obtained by calculating a truncated series based on these functions. However, Gram-Schmidt algorithm has some drawbacks such as numerical instability and high volume of computations. Here, to fix these flaws, we state the following Theorem in which the following notation are used.

$$
\begin{gathered}
\widehat{\boldsymbol{a}}=\left[\begin{array}{c}
\hat{a}_{1} \\
\hat{a}_{2} \\
\vdots \\
\hat{a}_{N}
\end{array}\right], \quad \overline{\boldsymbol{a}}=\left[\begin{array}{c}
\bar{a}_{1} \\
\bar{a}_{2} \\
\vdots \\
\bar{a}_{N}
\end{array}\right], \quad \overline{\boldsymbol{F}}=\left[\begin{array}{c}
\hat{f}_{1} \\
\hat{f}_{2} \\
\vdots \\
\hat{f}_{N}
\end{array}\right], \quad B=\left[\begin{array}{cccc}
\beta_{11} & 0 & & 0 \\
\beta_{21} & \beta_{22} & \cdots & 0 \\
& \vdots & \ddots & \vdots \\
\beta_{N 1} & \beta_{N 2} & \cdots & \beta_{N N}
\end{array}\right], \\
\Psi=\left[\begin{array}{ccccc}
\psi_{11} & \psi_{12} & & \psi_{1 N} \\
\psi_{21} & \psi_{22} & \cdots & \psi_{2 N} \\
& \vdots & \ddots & \vdots \\
\psi_{N 1} & \psi_{N 2} & \cdots & \psi_{N N}
\end{array}\right], \quad \widetilde{\Psi}=\left[\begin{array}{cccc}
\tilde{\psi}_{11} & \tilde{\psi}_{12} & & \tilde{\psi}_{1 N} \\
\tilde{\psi}_{21} & \tilde{\psi}_{22} & \cdots & \tilde{\psi}_{2 N} \\
& \vdots & \ddots & \vdots \\
\tilde{\psi}_{N 1} & \tilde{\psi}_{N 2} & \cdots & \tilde{\psi}_{N N}
\end{array}\right],
\end{gathered}
$$

where $\psi_{i j}=<\mathbb{L} \psi_{j}, \psi_{i}>, \tilde{\psi}_{i j}=<\mathbb{L} \psi_{j}, \tilde{\psi}_{i}>, \hat{f}_{i}=<f, \psi_{i}>, \beta_{i i}>0, \quad i, j=1, \ldots, N$. 
Theorem 4.Suppose that $\left\{\psi_{i}(x)\right\}_{i=1}^{\infty}$ a linearly independent set in $W_{2}^{4}[0,1]$ and $\left\{\tilde{\psi}_{i}(x)\right\}_{i=1}^{\infty}$ be a normalized orthogonal system in $W_{2}^{4}[0,1]$, such that $\tilde{\psi}_{i}(x)=\sum_{k=1}^{i} \beta_{i k} \psi_{k}(x)$. If $y(x)=$ $\sum_{i=1}^{\infty} \bar{a}_{i} \psi_{i}(x) \simeq y_{N}(x)=\sum_{i=1}^{N} \bar{a}_{i} \psi_{i}(x)=\sum_{i=1}^{N} \hat{a}_{i} \tilde{\psi}_{i}(x)$ then $\Psi \overline{\boldsymbol{a}}=\overline{\boldsymbol{F}}$.

Proof.Suppose that $y(x) \in W_{2}^{4}[0,1]$ then $y(x)=\sum_{i=1}^{\infty} \bar{a}_{i} \psi_{i}(x)=\sum_{i=1}^{\infty} \hat{a}_{i} \tilde{\psi}_{i}(x)$. Now, by truncating N-term of the two series, because of $y_{N}(x)=\sum_{i=1}^{N} \bar{a}_{i} \psi_{i}(x)=\sum_{i=1}^{N} \hat{a}_{i} \tilde{\psi}_{i}(x)$ and since $\tilde{\psi}_{i}(x)=\sum_{k=1}^{i} \beta_{i k} \psi_{k}(x)$ so

$$
\sum_{i=1}^{N} \bar{a}_{i} \psi_{i}(x)=\sum_{i=1}^{N} \hat{a}_{i} \tilde{\psi}_{i}(x)=\sum_{i=1}^{N} \hat{a}_{i}\left(\sum_{k=1}^{i} \beta_{i k} \psi_{k}(x)\right)=\sum_{k=1}^{N}\left(\sum_{i=k}^{N} \hat{a}_{i} \beta_{i k}\right) \psi_{k}(x) .
$$

Due to the linear independence of $\left\{\psi_{i}(x)\right\}_{i=1}^{\infty}, \bar{a}_{k}=\sum_{i=k}^{N} \hat{a}_{i} \beta_{i k}, k=1, \ldots, N$ therefore

$$
\overline{\boldsymbol{a}}=B^{T} \widehat{\boldsymbol{a}} .
$$

Eq. (7), imply $\mathbb{L} y_{N}(x)=f(x)$. For $i=1, \ldots, N$ we have

$$
\begin{aligned}
&<\mathbb{L} y_{N}, \tilde{\psi}_{i}>=<f, \tilde{\psi}_{i}> \Rightarrow \sum_{j=1}^{N} \hat{a}_{j}<\mathbb{L} \tilde{\psi}_{j}, \tilde{\psi}_{i}>=<f, \tilde{\psi}_{i}> \\
& \Rightarrow \sum_{j=1}^{N} \hat{a}_{j} \sum_{k=1}^{i} \beta_{i k} \sum_{l=1}^{j} \beta_{j l}<\mathbb{L} \psi_{l}, \psi_{k}>=\sum_{k=1}^{i} \beta_{i k}<f, \psi_{k}> \\
& \Rightarrow \sum_{j=1}^{N} \hat{a}_{j} \sum_{k=1}^{i} \sum_{l=1}^{j} \beta_{i k}<\mathbb{L} \psi_{l}, \psi_{k}>\beta_{l j}^{T}=\sum_{k=1}^{i} \beta_{i k}<f, \psi_{k}> \\
& \Rightarrow \sum_{j=1}^{N} \hat{a}_{j}\left(B \Psi B^{T}\right)_{i j}=\sum_{k=1}^{i} \beta_{i k}<f, \psi_{k}> \\
& \Rightarrow\left(B \Psi B^{T}\right) \widehat{\boldsymbol{a}}=B \overline{\boldsymbol{F}} .
\end{aligned}
$$

Eq. (8), imply $B \Psi \overline{\boldsymbol{a}}=B \overline{\boldsymbol{F}}$, hence

$$
\Psi \overline{\boldsymbol{a}}=\overline{\boldsymbol{F}} .
$$

It is necessary to mention that here we solve the system $\Psi \overline{\boldsymbol{a}}=\overline{\boldsymbol{F}}$ which obtained without using the Gram-Schmidt algorithm.

\section{Numerical examples}

To illustrate the effectiveness and the accuracy of the proposed method, two numerical examples are considered in this section. Figures 1,2,3 and 4 show that the approximate solution and its 
derivatives up to third-order, converge to the exact solution and its derivatives. We solved these examplesby the reproducing kernel Hilbert space method with $x_{i}=\frac{i-1}{N-1}, i=1, \ldots, N$ for $N=5$.

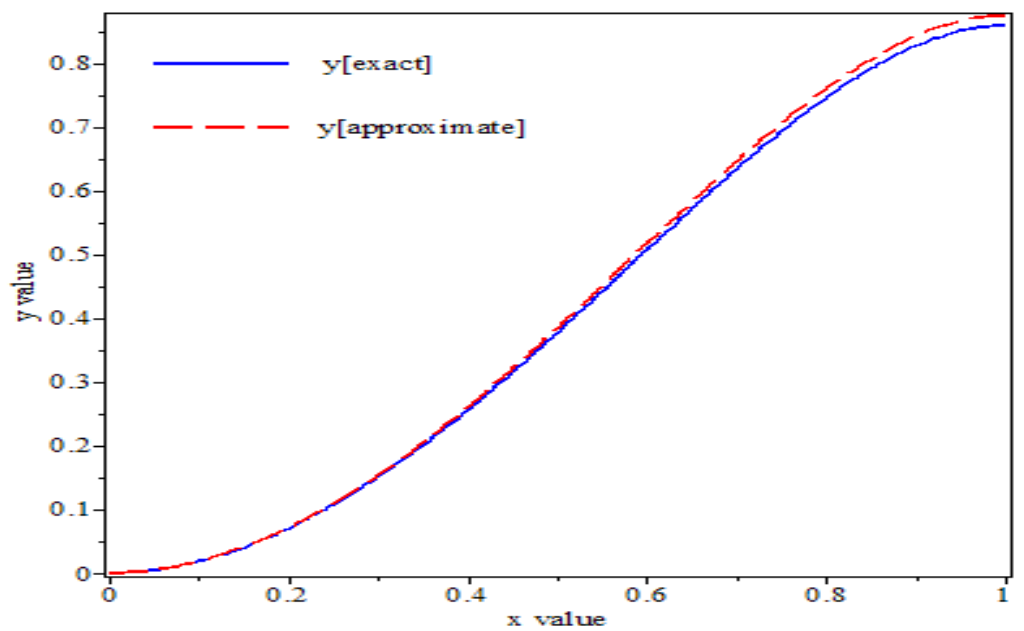

Figure 1: Comparison between approximate solution and the exact solution for Example 1 for $N=5$.

Example 1.We consider the following third-order BVP

$$
\left\{\begin{array}{cc}
\mathrm{y}^{\prime \prime \prime}(\mathrm{x})-\mathrm{xy}(\mathrm{x})=\left(\mathrm{x}^{3}-2 \mathrm{x}^{2}-5 \mathrm{x}-3\right) \mathrm{e}^{\mathrm{x}}, & 0 \leq \mathrm{x} \leq 1, \\
y(0)=0, & y^{\prime}(0)=1,
\end{array}\right.
$$

The exact solution of the above system is $y(x)=x(1-x) e^{x}$.

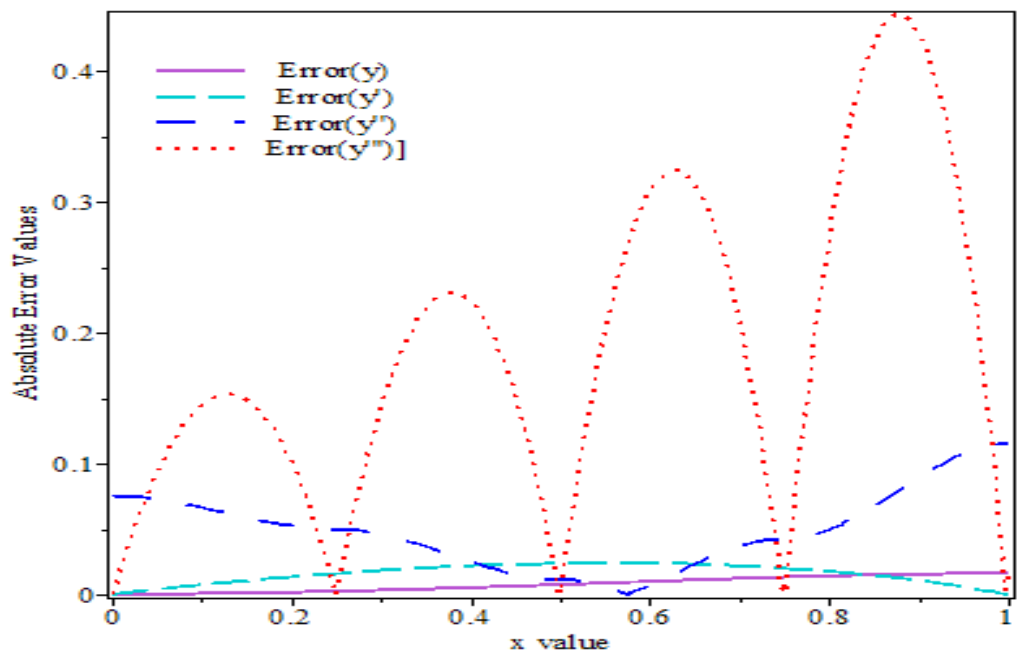

Figure 2: The absolute error between approximate solution and the exact solutionand its derivatives for Example 1 for $N=5$.

Example 2.Consider the following third-order BVP

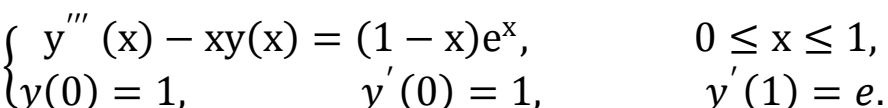


The exact solution of the above system is $y(x)=e^{x}$.

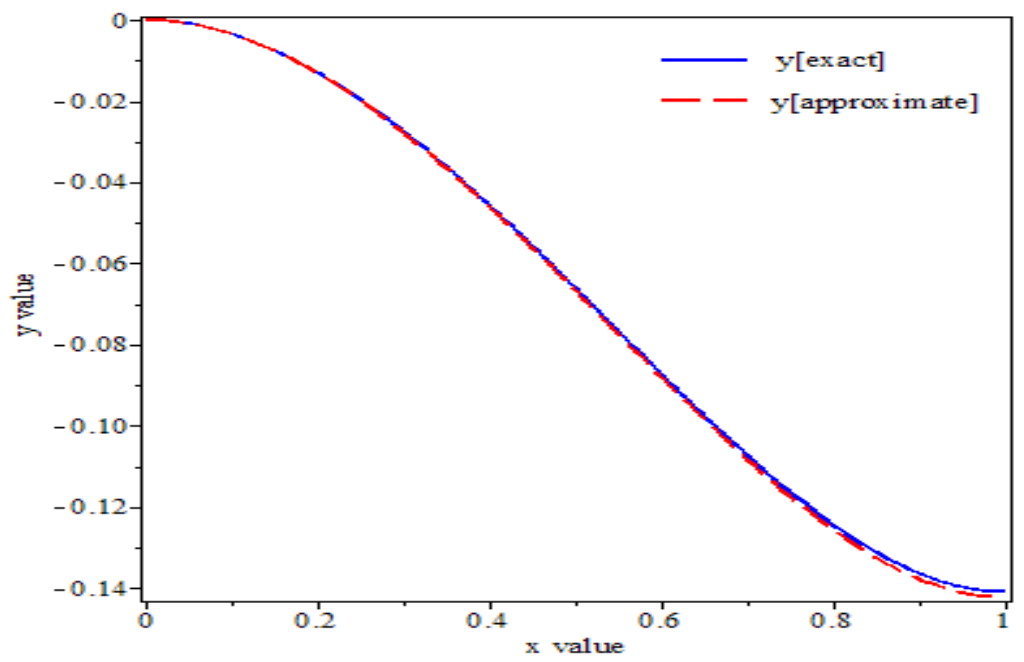

Figure 3: Comparison between approximate solution and the exact solution for Example 2 for $N=5$.

\section{Conclusions}

In this paper, we introduced the new implementation of reproducing kernel Hilbert space method to obtain the approximate solution to some third-order boundary value problems with variable coefficients. The reliability of the method and reduction of the amount of computation gives this method a wider applicability.The obtained numerical results confirm that the method is rapidly convergentand show that the approximate solutionconverge to the exact solution.

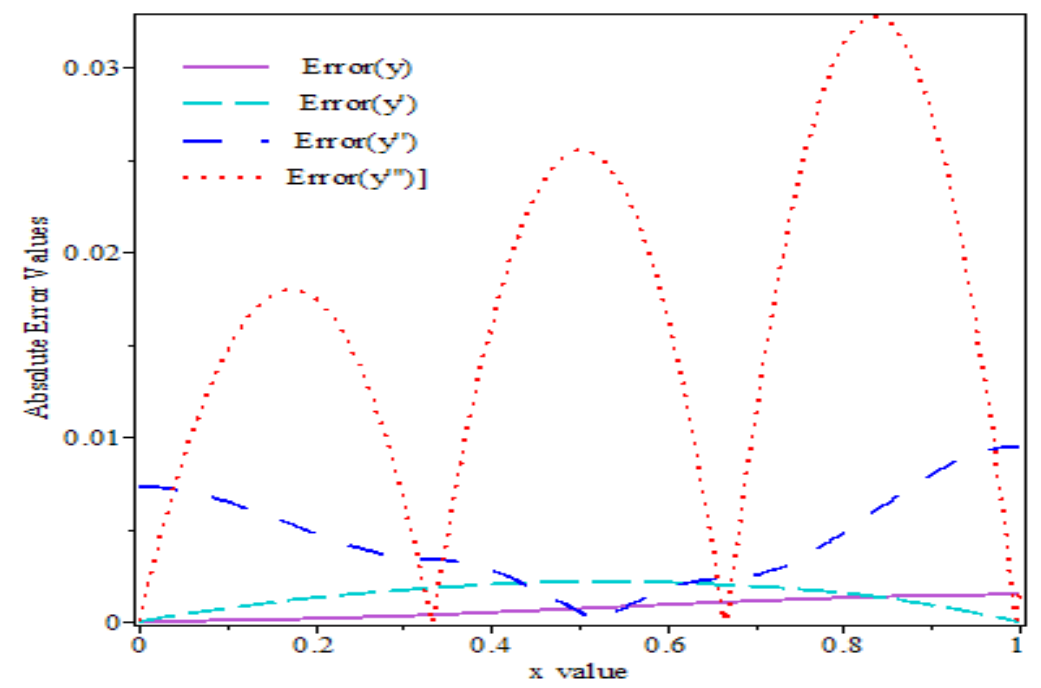

Figure 4: The absolute error between approximate solution and the exact solutionand its derivatives for Example 2 for $N=5$. 


\section{Apendix A.}

$$
\begin{aligned}
c_{1}=c_{2}=0, \quad c_{3} & =\frac{x^{2}}{7 !}\left(-14 x^{4}+3 x^{5}+45372-30262 x+21 x^{3}\right), \quad c_{4} \\
& =-\frac{x^{2}}{7 !}\left(2 x^{5}+30262-20212 x-7 x^{4}+35 x^{2}\right), \\
& c_{5}=c_{6}=0, \quad c_{7}=\frac{x(x-1)^{2}}{6 !}, \quad c_{8}=-\frac{(2 x+1)(x-1)^{2}}{7 !}, d_{1}=-\frac{x^{7}}{7 !}, d_{2}=\frac{x^{6}}{6 !}, d_{3} \\
& =\frac{x^{2}}{7 !}\left(-14 x^{4}+3 x^{5}+45372-30262 x\right), \quad d_{4} \\
& =-\frac{x^{2}}{7 !}\left(2 x^{5}+30262-20212 x-7 x^{4}\right), d_{5}=-\frac{x^{3}}{3 ! 4 !}, d_{6}=\frac{x^{2}}{2 ! 5 !}, d_{7} \\
& =\frac{x^{2}(x-2)}{6 !}, \quad d_{8}=\frac{x^{2}(3-2 x)}{7 !} .
\end{aligned}
$$

\section{References}

[1] F. Geng, M. Cui, A reproducing kernel method for solving nonlocal fractional boundary value problems, Appl. Math. Letters 25 (2012) 818-823.

[2] F. Geng, S. Qian, Reproducing kernel method for singularly perturbed turning point problems having twin boundary layers, Appl. Math. Letters 26 (2013) 998-1004.

[3] H. Du, M. Cui, Approximate solution of the Fredholm integral equation of the first kind in a reproducing kernel space, Appl. Math. Letters 21 (2008) 617-623.

[4] H. Du, M. Cui, Representation of the exact solution and stability analysis on the Fredholm integral equation of the first kind in a reproducing kernel space, Appl. Math. Comp. 182 (2006) 1608-1614.

[5] L. Yang, J. Shen, Y. Wang, The reproducing kernel method for solving the system of linear Volterra integral equations with variable coefficients, J. Comp. Appl. Math 236 (2012) 23982405.

[6] Z. Chen, W. Jiang, The exact solution of a class of Volterra integral equation with weakly singular kernel, Appl. Math. Comp. 217 (2011) 7515-7519.

[7] Z. Chen, Y. Lin, The exact solution of a class of linear integral equation with weakly singular kernel, J. Math. Anal. Appl. 344 (2008) 726-736.

[8] W. Jiang, M. Cui, The exact solution and stability analysis for integral equation of third or frist kind with singular kernel, Appl. Math. Comp. 202 (2008) 666-674.

[9] F. Geng, Solving integral equation of the third kind in the reproducing kernel space,Bulltein Iranian Math. Society 201 (2011) 1-9.

[10] M. Cui, Z. Deng, On the best operator of interpolation, J. Math. Numerica.Sinica. 8(2) 1986 209-216.

[11] M. Cui, F. Geng, Solving singular two-point boundary value problem in reproducing kernel space, J. Comp. Appl. Math. 205 (2007) 6-15.

[12] W. Wang, M. Cui, Bo Han, A new method for solving a class of singular two-point boundary value problems, Appl. Math. Comp. 206 (2008) 721-727. 
[13] F. Geng, M. Cui, Solving singular nonlinear two-point boundary value problems in the reproducing kernel space, J. of the Korean Math. Society 45 (3) (2008), 631-644.

[14] F. Geng, M. Cui, Solving singular nonlinear second-order periodic boundary value problems in the reproducing kernel space, Appl. Math. Comp. 192 (2007), 389-398.

[15] W. Jiang, M. Cui, Y. Lin, Anti-periodic solutions for Rayleigh-type equations via the reproducing kernel Hilbert space method, Commun Nonlinear SciNumerSimulat 15 (2010) 1754-1758.

[16] E. Babolian, Sh. Javadi and E. Moradi, RKM for the Numerical Solution ofBratu's Initial Value Problem, submitted on December 27, 2013.

[17] F. Geng, Iterative reproducing kernel method for a beam equation with third-order nonlinear boundary conditions, Mathematical Sciences 2012, 6:1.

[18] B. Wu, X. Li, Application of reproducing kernel method to third order three-point boundary value problems, Appl. Math. Comp. 217 (2010) 3425-3428.

[19] F. Geng, M. Cui, Solving a nonlinear system of second order boundary value problems, J. Math. Anal. Appl. 327 (2007), 1167-1181.

[20] Gh. Akram, H. Rehman, Solution of fifth order boundary value problems in reproducing kernel space, Middle-East Journal of Scientific Research 10(2) 2011, 191-195.

[21] M. Mohammadi, R. Mokhtari, Solving the generalized regularized long wave equation on the basis of a reproducing kernel space, J. Comp. Appl. Math. 235 (2011) 4003-4014.

[22] M. Cui, Y. Lin, Nonlinear numerical analysis in reproducing kernel Hilbert space, Nova Science Publisher, New York, 2009.

[23] M. Rabbani,New Homotopy Perturbation Method to Solve Non-Linear Problems, Journal of mathematics and computer Science 7 (2013) 272 - 275.

[24] M. Saravi, A. Nikkar, M. Hermann, J. Vahidi, R. Ahari, A New Modified Approach for solving seven-order Sawada-Kotara equations, Journal of mathematics and computer Science 6 (2013) 230-237.

[25] S.Gh. Hosseini, S.M. Hosseini, M. Heydari, M. Amini,The analytical solution of singularly perturbed boundary value problems, Journal of mathematics and computer science 10 (2014), 722. 